P. Berander and C. Wohlin, "Identification of Key Factors in Software Process Management - A Case Study", Proceedings International Symposium on Empirical Software Engineering, pp. 316-325, Rome, Italy, 2003. 


\title{
Identification of Key Factors in Software Process Management - A Case Study
}

\author{
Patrik Berander and Claes Wohlin \\ Department of Software Engineering and Computer Science \\ Blekinge Institute of Technology, PO Box 520, S-372 25 Ronneby SWEDEN \\ Patrik.Berander@bth.se,Claes.Wohlin@bth.se
}

\begin{abstract}
When conducting process related work within an organization, it is important to be aware of which factors that are most important to consider. This paper presents an empirical case study that was performed in order to find the key success factors in process management. One factor, namely synchronization of processes, was considered as much more important within the studied organization than within the studied literature. This shows that more research might be needed in this area. The study further shows that it is important to relate process improvement work to the properties of the affected organization and that the key factors identified are highly interrelated.
\end{abstract}

\section{Introduction}

According to Marciniak [1], the most serious problems in software organizations typically concern organizational procedures and cultural behavior. These problems are not something individuals within the organization generally can fix themselves. Therefore, a comprehensive and longterm focus on the software process is required to solve them [1]. Further, to compete well in today's marketplace, it is a pre-condition to have best-practice engineering standards in place, measuring the conformance, and continually trying to improve [2].

Despite the need of focusing on the processes, users of processes often have an inner resistance against defined processes and the change of processes [3]. It is also common that the defined processes are disregarded or deviated from (e.g. [4], [5]). To solve these problems, knowledge about important factors to consider is required. One of the reasons for having defined processes is that the organization could share knowledge gained [6]. Therefore, it seems obvious that people working with processes should reuse the knowledge others have gained in process related work.

This paper presents a case study where the key factors for successful management and evolution of the software process were studied. The study was launched as it was viewed as essential to identify key success factors for managing the software process before introducing an improvement programme. These are found through an empirical case study involving three parts: a qualitative part (interviews), literature part (survey), and a quantitative part (questionnaire).

The remainder of this paper is structured as follows. Section 2 outlines how the empirical study was performed. Section 3 presents the results that were obtained within each part of the study. In Section 4, a combined analysis of the three parts is presented and the factors found are ranked in relation to each other. Section 5 discusses the implications of the findings and what conclusions that could be drawn. Finally, Section 6 concludes the results and discusses further work within the area.

\section{Method}

The objective of this paper is to identify key factors for managing the software process. The factors are identified through method triangulation, i.e. the use of several different approaches to identify the key factors. Moreover, the intention is to study whether the factors identified in a specific software developing organization also are the factors that are found in literature. The triangulation is done by combining the three parts of the empirical study to retrieve one overall result.

The three different parts constituting the overall study were done and combined as follows. First, a number of interviews were conducted and the key factors for software process management, according to these interviews, were identified. Second, a literature survey was done and key factors for successful software process management and improvement according to literature were identified. Finally, a questionnaire was used to capture the viewpoint of the whole organization. The questionnaire was based on the identified factors from the interviews and the literature survey. Finally, the results were combined by identifying the most important factors from the questionnaire and 
relating that to the findings with respect to these factors in the interviews and the literature survey respectively. This results in the identification of a set of key factors for software process management.

Before discussing the individual parts in the study, the research setting, i.e. the organization studied, is described briefly in Section 2.1. The different parts of the study are then presented in the following three sections with the interviews in Section 2.3, the literature survey in Section 2.4 and the questionnaire in Section 2.5. Finally, the validation using method triangulation is further discussed in Section 2.6.

\subsection{Research Settings}

This paper is based on an investigation that was performed at a software development organization in the south of Sweden. At the time of the study, the organization had about 400 employees that were working in different software development projects (primarily development of realtime systems within the communication business domain). Such projects typically include 60-120 persons for 12-18 months.

The defined processes in the organization were mostly developed within each department or unit (e.g. a design unit had a design process of its own) and the organization also had some standard processes that all departments should follow (e.g. a process for inspecting artifacts). In addition, the company followed an internally developed project management model.

\subsection{Goal of Empirical Study}

The initial goal of the empirical study was to get an understanding of the processes in the studied organization. The intention was to investigate how people perceived the defined processes and to do a mapping of which defined processes that are useful and what problems that are encountered in others. It was also the aim to assess how mature the processes were in the organization. A side effect of this work was that several factors were identified as key factors in the organization. Due to that the design was not primarily intended to find such factors, the six identified key factors were never compared with each other in the questionnaire.

\subsection{Qualitative Part (Interviews)}

The interviews focused on one part of the software process, namely the requirements engineering process. The interviews were part of a project aiming at evaluating the current requirements engineering process and identifying potential improvements. In the interviews, the objective was to capture the tacit knowledge about the processes that resides with the co-workers.

The interview questions were rather open and there were much room for discussions about the factors that were mentioned. 25 persons from all operative departments within the organization were interviewed. Three persons were part of the team that conducted the interviews: one employee within the organization, one external consultant, and one researcher.

\subsection{Literature Part (Survey)}

A detailed literature survey was conducted after the interviews. The main reason for this was that both researchers have general knowledge in the area of process management and performing the literature survey prior to the interviews could lead to that we were "fishing" for the key factors identified in literature. The general knowledge about processes implied that no preconceived thoughts about exactly which factors that were key factors were present. The intention was that the interviews should capture the viewpoints of the personnel and hence the discussions should not be tainted by the factors that can be found in the literature.

In addition, the literature survey was performed after the interviews for two reasons. First, it was possible to see whether the problems mentioned in the interviews also were mentioned in the literature about processes. Second, it was possible to find out if there were additional concerns mentioned in the literature that were not mentioned in the interviews.

The search criteria that were used in the literature survey were based on the interviews as well as the general knowledge in processes by the researchers. This preknowledge resulted in that additional factors were found in the literature.

\subsection{Quantitative Part (Questionnaire)}

The objective of the questionnaire was to obtain a larger sample and also a more representative sample for the whole organization than in the interviews. The questionnaire was formulated based on the findings in the interviews and the additional information obtained from the literature survey.

The questionnaire comprised of 23 questions, plus demographics and one open-ended question. The first 18 questions were multiple-choice questions. Questions 19-23 were weighted questions where the respondents had the possibility to rank their answers. In addition to ordinary ranking, they had the possibility to put weights on their answers by apportioning 100 points between the answering-alternatives [7]. For example, alternative A gets 30 
points, B 20 points, and C 50 points. With this result, alternative $C$ stands for 50 percent of the value, alternative $B$ for 20 percent and so on. This made it possible to see how much more important some factors were than others (in contrast to ordinary ranking where you only could see that one alternative is more important than another).

The questionnaire was sent out to 84 persons in the organization (not the same persons as in the interviews). 65 persons answered the questionnaire, which is equivalent to 17 percent of the organization (software development part), and 77 percent of the sample. This sample was symmetrically distributed both vertically (i.e. management levels) and horizontally (e.g. departments, units).

\subsection{Validation of Results}

To avoid misinterpretations in the questionnaire, three persons reviewed the questions. Further, a pilot test was performed with three respondents to test the questionnaire. This test resulted in some minor adjustments of some questions and the order of the questions.

As mentioned, the objective was to ensure internal validity of the obtained results through triangulation. Two different types of triangulation have been used. First, analyst triangulation [8] was used to verify that the results in the interviews were interpreted correctly. In this triangulation, the three interviewers discussed their individual results and reached consensus about the findings. Further, methods triangulation [8] was used between the three conducted parts of the study, as presented in Figure 1 (the arrows represents validation). This triangulation compared the results in order to validate that the result from each study corresponds to the other studies.

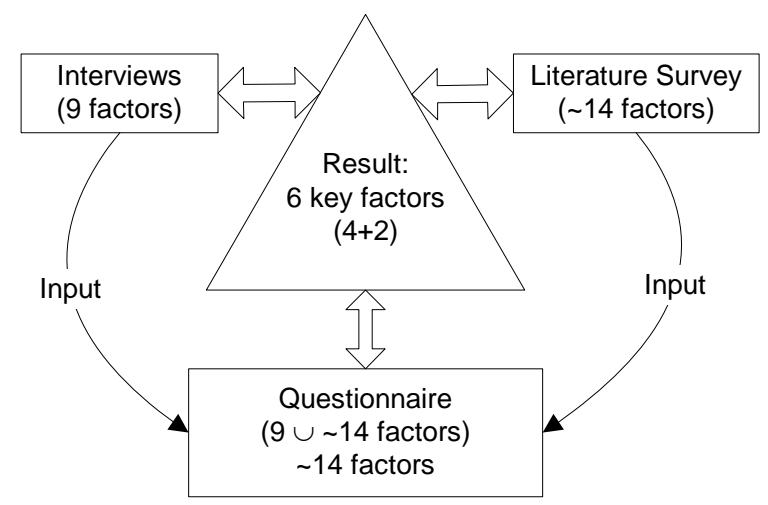

Figure 1. Methods Triangulation of Results

\section{Result}

In this section, the results of the three parts of the study are presented. However, not all factors that were mentioned in the literature and the two empirical parts are pre- sented here. Many more factors were discussed (e.g. training, goal setting, measurements, process staffing) but in order to keep the focus on the vital few, i.e. the key factors, only the factors being viewed as most important in the study are presented.

As can be seen in Figure 1, the interviews and the literature survey served as input to the questionnaire. The interviews explicitly found nine important factors. From the literature survey, it is possible to generate a long list of important factors (depending how they are counted) and an approximate number is around 14 factors. However, only factors that were found in several books or articles were included. When designing the questionnaire, the union of these two sources was used as a foundation. This resulted in approximately 14 factors (depending how they are counted) that were considered in the questionnaire. Hence any factor included in the questionnaire was either viewed as important in the interviews or in literature, or in both.

Based on the result from the three parts, four factors identified in the interviews and two factors identified in the literature were viewed as being key factors. The criteria for considering a factor as a key factor lies in the frequency and to what extent the factor was discussed in each part of the study, and that it was considered important in at least two parts. In the interviews and the literature part, the identified key factors were those that were mentioned the most, and mostly discussed. In the questionnaire it was those that were highest ranked.

In the listing below, it is in parenthesis indicated whether the factor initially were identified in the interviews (part 1) or the literature survey (part 2):

- Change management (part 1), this factor is related to the necessity in keeping the defined process under configuration control and ensure that it is updated and is in accordance with the actual work.

- Synchronization of process parts (part 1), the process steps must be synchronized so that exit criteria, including documents, are consistent to entry criteria in a following step.

- Baselining the current way of working (part 1), process descriptions should be derived bottom-up, i.e. they should be based on the current way of working.

- Documentation (part 1), the documentation (e.g. granularity level, location) of the process was identified as one factor.

- User involvement (part 2), the actual users of the process must be involved in the definition of a process. This is partially related to baselining the current way of working. However, it also includes that the users should be involved in the changes of a defined process. 
- Management commitment (part 2), management must provide money and resources to show their commitment and that process management is important.

Next, the support for these six factors in the three parts of the study is discussed.

\subsection{Qualitative Part (Interviews)}

Almost all of the respondents in the interviews expressed that they are in need of usable process descriptions. Several respondents felt that they did not see the whole picture of the development process, and hence they could not see what their contributions were. The impact was that motivation could suffer and they did not have a real commitment towards their work. Several factors were mentioned as areas where today's process handling could be improved. In particular, the findings with respect to the six key factors are as follows.

3.1.1. Change Management. The respondents argued that process descriptions often are made and then forgotten and not updated. As a side effect, the descriptions are not always used since they often do not reflect the current way of working but rather as the organization has worked in the past.

3.1.2. Synchronization. The respondents argued that synchronization and integration between different processes is a must (e.g. synchronization between design/implementation and test processes). The respondents seemed to find it hard to see a continuum in the overall process because defined processes were not synchronized and integrated. Unsynchronized processes could for example mean that a first phase has $\mathrm{x}$ as exit criteria while a following phase has $\mathrm{x}$ and $\mathrm{y}$ as entry criteria, which results in the second phase having problems starting its work (due to the lack of y).

\subsubsection{Baselining the Current Way of Working. Baselin-} ing the current way of working was one of the factors most mentioned. Respondents argued that defined processes were "forced" on them and they had no active part in defining the process. This often meant that the process descriptions were not suited for their real working environment but rather were considered as an ideal picture. Hence, they were often neglected. Further, several respondents argued that the most urgent measure was to describe and baseline current work procedures; no improvement initiatives would have any effect until this was done. One respondent expressed himself rather clear: "The processes MUST be anchored in the real way of working”.
3.1.4. Documentation. Some respondents argued that it is important that process descriptions have the right level of detail. Often, the process descriptions were either too detailed (people stop thinking) and too specific to reuse, or too fuzzy, so that they had to be so much tailored for specific needs that they were not usable without much further work. The respondents argued that processes must be easy to adapt and be dynamic to suit different circumstances. Further, the respondents argued that the defined processes were too extensive for people to take their time to read them and there are too many descriptions of each process.

The main reasons for why the process descriptions were not followed were that they were hard to find, hard to understand and they did not fit the purpose, they argued. The essence of this part is what one of the respondents said: "The process must be an aid in the work instead of a load”.

3.1.5. User Involvement. The respondents of the interviews did not mention the involvement of users explicitly. However, this factor was hidden in other factors and several times it was implicitly expressed that they did not think that the future users of processes were enough involved in the development of defined processes (e.g. in baselining the current way of working).

3.1.6. Management Commitment. Management commitment was not mentioned explicitly in the study. Nevertheless, this factor was hidden in other factors (e.g. baselining, change management) and several respondents argued implicitly that they did not think that management gave them enough time and resources to improve their way of working.

\subsection{Literature Part (Survey)}

Based on the interviews, a literature survey was conducted to find the most important factors in process management, according to the literature in the field. Many factors were found in this survey. However, the discussion below presents only those six factors that were concerned as key factors in the overall study.

3.2.1. Change Management. The problem with change management occurs when the way of working changes but not the written process description. Conradi and Dybå [4] report that none of the twelve companies they investigated had any routines for updating defined processes. Curtis et al. [5] further argue that deviations from defined processes often originate from the lack of updating of the defined processes. Gilb [2] also aligns to these statements and argues that defined processes must not be static when there is better know-how in the organization. 
3.2.2. Synchronization. Synchronization was not a frequently mentioned factor in the literature. However, Humphrey [9] argues that the focus should be put on defining what input and output that are expected from a process/ phase. Pfleeger [6] also aligns to this and states that a defined process could require design before coding but allow many different design techniques to be used. This means that the input and output of design and coding are defined, but coding does not care of how the design was developed. Further, some work has been done about synchronization of more formal processes. However, the company investigated does not have very formal process descriptions and they do not intend to introduce more formal processes than the current.

\subsubsection{Baselining the Current Way of Working. The}

majority of models that handles process improvement discuss the importance of baselining the current way of working (e.g. [10], [11], [12]). This means that the process description describes the current way of working (descriptively) rather than how the employees are supposed to work (prescriptively). This implies that the risk of describing an ideal process (which is a common reason for deviations) is reduced.

3.2.4. Documentation. Researchers within the field seem to agree that the documentation should be easy to read, and not too formalistic but rather useful and necessary (e.g. [2], [4], [13], [14]). The main reason is that no matter how good the defined process is, people do not consider using it if they cannot understand the documentation or if it is too extensive.

The level of detail of a defined process should much depend on the people who will use the documents and how complex the task is [15]. Less experienced people need a lower abstraction level than more experienced people (e.g. [4], [5]). Further, the level of detail also affects the level of formalism introduced in the defined process; a higher abstraction level provides more freedom to own decisions and creativity. Therefore, different levels of detail are desirable to satisfy the needs of all the staff [5].

One important thing to remember is that defined processes shall impose consistency and structure. Defined processes could be very flexible when the level of detail is right. Steltzer and Mellis [16] ranked tailoring processes as the fourth most important out of ten success factors, based on a study performed at 56 software companies.

3.2.5. User Involvement. Steltzer and Mellis [16] ranked user involvement factor as number two out of the ten most important success factors (for process improvement). Besides this study, several authors discuss the importance of user involvement (e.g. [9], [14]). User involvement is primarily needed for two reasons. First, their first hand experience of the processes is valuable in the development (i.e. they know which part that is in most need of development/improvement). Second, participation reduces the resistance to the developed processes [16].

3.2.6. Management Commitment. Management Commitment was ranked as number one of the ten factors Steltzer and Mellis [16] ranked. Further, several authors discuss management commitment as very important (e.g. [17], [18]). The essence of management commitment is that management must provide time and resources for the staff if process initiatives should be successful. Management should also show that they believe in the initiative.

As described above, the material from the interviews and the literature survey was used as input to the design of the questionnaire. The results are presented below.

\subsection{Quantitative Part (Questionnaire)}

23 questions were asked in the questionnaire. Due to the large amount of data, it is not possible to provide the result of each individual question here. However, a general discussion that summarizes the answers relating to each key factor is provided. As indicated in Section 2.2, the initial intention of the study was not to identify these key factors, and hence the factors were not compared directly to each other. This means that no statistical test can be performed.

3.3.1. Change Management. A question about change management showed that 66 percent felt that process descriptions were updated too seldom or never. At the same time 25 percent felt that they were updated as they should while 9 percent thought that they were updated too often. Change management was also ranked higher than inconsistent process descriptions but was ranked lower than synchronization and baselines in one of the weighted questions.

3.3.2. Synchronization. When the respondents got the question if different departments/units had an understanding for each other's work, 63 percent answered that they had not. Further, when they answered how well they thought that processes were synchronized, 41 percent answered bad (33\%) or very bad (8\%). 50 percent did answer that the processes "could be better" synchronized while only 9 percent answered that they were "good". None of the respondents answered "very good" to this question.

This result is also shown in the answer to which factors that the respondents regarded as most important in a work description/process, where "workflow" (with entry and exit criteria for documents, artifacts etc.) was regarded as 
the single most important factor. This indicates that it was in the interfaces between processes the problems were located.

When the respondents got the question what they saw as the largest threats against successful process improvement they clearly indicated that "Processes are not synchronized between departments" was the most threatening factor. "Synchronization between processes" was also ranked as the most urgent problem to resolve.

Nevertheless, when the respondents were given the question if they discuss process-related faults with the people that are affected, 21 percent answered “always”, 34 percent answered "often" while 29 percent answered "sometimes".

\subsubsection{Baselining the Current Way of Working. When}

the respondents were asked to rank the urgency between seven problems they had encountered, they ranked "understand and document our current way of working" as the second most important factor, slightly after "synchronization between processes". When the respondents were asked who they believed were the most active users of process descriptions, 17 percent answered "staff" while the rest answered "quality persons" (27\%) or "management" (56\%). This indicates that the defined processes are not a baseline to the work environment of the staff, but rather to management or quality persons’ work.

3.3.4. Documentation. To the question "how detailed are process descriptions today?”, 37 percent answered that the descriptions had an adequate level, 42 percent answered that it depended on which description it was while "too general" (16\%) was a more common answer than "too detailed" (5\%). This result shows that 63 percent of the respondents thinks that the documentation of processes could be improved, at least some of the descriptions. "Inconsistent process descriptions" was ranked after synchronization, change management, and user involvement to the question of how the relative threats against successful process improvement were divided. Further, tailoring was ranked as the third most important factor of which problem that was the most urgent to resolve.

3.3.5. User Involvement. To the question "Who develops processes and process descriptions today?”, 50 percent answered that the staff were not involved, 8 percent did not know and 42 percent stated that the users were involved. To the follow-up question: "According to your opinion, who should develop processes and process descriptions?” 92 percent did answer that the users should be involved. Further, when the respondents were asked about the most important factors in process improvement, "user involvement” was ranked as the most important.
3.3.6. Management Commitment. The respondents to the questionnaire generally felt (66\%) that they did not have enough time devoted to develop structured solutions to problems. Further, 52 percent stated that they wanted to devote more or much more time to improvement activities while 37 percent wanted to devote the same amount of time and 11 percent wanted to devote less (8\%), much less $(0 \%)$, or no time $(3 \%)$. When the respondents were asked about the most important factors in process improvement, they ranked "management commitment" as the second most important.

\section{Combined Analysis}

In the discussion below, the results that were obtained when triangulating the three studies are presented. Hence, the discussion focuses on relating the findings from each of the studies to each other.

\subsection{Change Management}

The literature states that change management of defined processes is a key issue in process management. In both the interviews and the questionnaire, the respondents argued that process descriptions were updated too seldom. Further, the descriptions were not updated when the actual work methods were improved. Hence, they did not reflect the actual way of working. A good change management process should also facilitate the possibility to manage a process baseline [19] as discussed in Section 4.3.

\subsection{Synchronization}

The studied literature did not discuss synchronization very much even if some authors discuss the area to a certain extent. However, the interviews indicated that synchronization of processes was a problem within the organization. This was validated through the questionnaire where 91 percent answered that they were not satisfied with the synchronization of processes. This indicates that it is one of the largest problems in the studied organization. Further, one respondent in the open-ended question in the questionnaire argued that changes were made without consideration of the stakeholders in the process and in connected processes.

Respondents in the questionnaire answered that they discussed process related problems with other people. It seems like these discussions do only relate to issues that are specific for the process/department. This indicates that people generally are good at communicating within the departments, but they are not very good at discussing with people from other departments or processes. Kock [20] states that many problems are divided between several 
departments/processes and hence the interaction between departments/processes must be improved in order to gain the knowledge that are possessed by all involved parties.

\subsection{Baselining the Current Way of Working}

In the literature, baselining the process (descriptive modeling) is discussed as a key activity in process management [21]. The people in the investigated organization seem to support this statement, in both the interviews and the questionnaire. Further, people in the interviews and the open-ended question in the questionnaire, argued that defined processes were developed without involvement from the staff and without having the process well anchored in reality. The respondents of the questionnaire support this when they answered that the staff should be more involved in the development of processes. This issue is therefore tightly connected to the issue of user involvement in Section 4.5.

\subsection{Documentation}

Documentation was often mentioned as a problem in the interviews. The questionnaire showed that the "inconsistent process descriptions" was ranked after change management, synchronization, and user involvement as threat to successful process management. However, a rather large part of the respondents answered that the quality of the documents varied between different descriptions. The literature also discusses documentation as a problem; most often, the documentation is too extensive without a reason (e.g. [2], [13]). Adaptation (tailoring) of processes was discussed and considered as important during the interviews, in the literature survey, and it was also rather highly ranked in the questionnaire (after the already proven important factors: synchronization/interfaces between processes/ departments, and baselines).

\subsection{User Involvement}

According to the questionnaire, the wrong persons develop the process descriptions in the investigated organization. The answers indicate that most people do not consider the staff as being a part of the development, but the majority of the respondents think the staff should be a part of this activity. These figures clearly indicate that the staff wants to be a more active part in the development of defined processes. One respondent that answered the openended question, further strengthens this argumentation when he talks about the people developing processes at present: "these developers do not have an understanding of how people work in reality“, which means that if usable process descriptions should be developed, the affected staff should be a part of the development. The issue of having affected users involved is also discussed much in the literature, and was also mentioned implicitly in the interviews at a few occasions.

\subsection{Management Commitment}

Management commitment was a factor that was highly ranked in the questionnaire as well as in the literature studied. Further, one respondent argued in the open-ended question of the questionnaire that management tried to shortcut the process whenever a project became critical. He further argued that it would be nice to hear that when a project becomes critical it is the most important time to follow processes; if management does not believe in the processes, their staff will not.

People in the interviews did not mention management commitment explicitly. However, time was mentioned several times in relation to other tasks. They argued that they did not get the time they wanted to improve their work but all their time was assigned to projects that produced products.

The questionnaire clearly indicated that not enough time is devoted to structured solutions to problems. This shows that the staff really wants to devote time to process activities, but management does not provide the right amount of time and resources.

\subsection{Validity}

The internal validity (i.e. the validity of the results within the organization) of the presented study is addressed by triangulating (see 2.6) three different sources of information: interviews, a literature survey and a questionnaire.

Moreover, the rather large sample size and an even spread across the organization help ensuring the internal validity. The results from the study has also been presented and discussed in-depth in the organization to validate that the results are interpreted correctly.

The external validity (i.e. the degree of generalization) from a case study is much more difficult to determine. However, it should be noted that most of the success factors identified in the organization were supported by the literature, which indicates that the results are not specific for the organization. Moreover, the size of the organization and that it delivers products on a world market (in contrast to a small domestic consultant company) at least indicate that it is not likely that the findings are valid for this organization only. However, it is hard to determine to what extent the findings are possible to generalize. 


\subsection{Factor Importance}

The next step is to see how important each factor was in each of the three parts of the study. Here, an attempt has been made to quantify the importance of the factors. The importance of the factors in the questionnaire was relatively easy to rank because weights were used. The factors in the literature survey and the interviews were harder to rank. Therefore, pair-wise comparisons between all factors in each part of the study were performed, which made it easier to compare the factors than through a direct ranking.

When doing the pair-wise comparisons, different criteria were used as determination of which factor that was most important. In the interviews, the number of times the factor was mentioned and how thoroughly it was discussed were used as criteria. In the literature study, the number of articles found that deal with the factor, how they perceived the factor, and to what extent it was examined were used as criteria. In the questionnaire, the weights and answers provided in the questionnaire were used as criteria.

The researchers performed the comparison and it could look rather subjective at a first glance. However, the quantitative results were rather clear and unambiguous due to that the respondents set the weights themselves. The qualitative results are also seen as rather reliable due to the analysts triangulation (see section 2.6) and to the half-year experience at the organization investigated. The factors in the literature survey were the hardest to compare. However, with the study of Steltzer and Mellis as a starting point and with an extensive study according to the criteria mentioned above, the result of this ranking could also be seen as rather reliable. Further, both researchers have some experience in the area of process management, which made the comparisons of "literature factors" easier.

The result became as shown in Table 1, where the lowest numbers are considered as most important. The discussion about the implications of this result is presented in Section 5.

\section{Discussion}

As seen in Table 1, baseline and synchronize the software process is considered as the two most important factors when adding the three parts of the study together. To provide a baseline is considered rather important in all three studies and becomes therefore important overall. Synchronization, on the other hand, is ranked as the most important factor in both organization-specific parts of the study but is ranked lowest in the literature part.

User involvement and management commitment are ranked as third and fourth important respectively. These two were ranked highest in the literature survey but ranked lowest in the interviews. However, user involvement was ranked second in the questionnaire and management commitment fourth in the questionnaire, which must be seen as they still are very important within the organization. Both these might have been higher ranked if they had been addressed more directly in the interviews. This was a negative thing when not "fishing" (see 2.4) for the results. On the other hand, factors like synchronization might not have been mentioned in the interviews if "fishing".

Regarding change management, the results of each part of the study corresponded very well with each other. All three parts of the study ranked the change management issue as the fourth or fifth highest ranked. Still, 75 percent of the respondents argued that the defined processes were not updated as they should.

Documentation was ranked third in the interviews but was ranked as 5 and 6 in the literature survey and the questionnaire respectively. This shows that even if the organization have some problems with documenting the processes, it is not as important as other issues. Still, the majority is not satisfied with the documentation as it is today.

Overall, the result of the three studies aligns rather well with each other. The rankings between literature and this particular organization are different but that is not very surprising. The big difference lies in the issue of synchronization. Synchronization is ranked highest in the organization-

Table 1. Total score for the different factors when combining the studies.

\begin{tabular}{|l|l|l|l|l|}
\hline Factor & Qualitative & Quantitative & Literature & Total \\
\hline 1. Baselining & 2 & 3 & 3 & $\mathbf{8}$ \\
\hline 1. Synchronization & 1 & 1 & 6 & $\mathbf{8}$ \\
\hline 3. User Involvement & 5 & 2 & 2 & $\mathbf{9}$ \\
\hline 4. Management Commitment & 6 & 4 & 1 & $\mathbf{1 1}$ \\
\hline 5. Change Management & 4 & 5 & 4 & $\mathbf{1 3}$ \\
\hline 6. Documentation & 3 & 6 & 5 & $\mathbf{1 4}$ \\
\hline
\end{tabular}


specific part while lowest in the literature part. This is, according to us, a very interesting result. The major question is if this is something that is organization specific or this is a common issue in other organizations as well. Organizations with the same properties (e.g. size, organizational structure) might be affected by the same problems while it might not be as evident in smaller organizations.

The difference between the organization and the literature might be an indication that further studies ought to be conducted and that the subject of synchronization is not well enough discussed in industry and the research community. The difference between the literature and the conducted study could origin in that the literature is based on the voice of the theories of management literature while the study is based on the voice of the practitioners.

The results further indicate that it is very important that the people that perform process improvements are well aware of which problems an organization have. In some organizations, documenting the processes might be the largest problem. However, in this organization it was considered as the least important problem (of the key factors). Remember that management commitment was considered as the most important in the study performed by Steltzer and Mellis [16] but was passed by user involvement in this study. This means that we cannot draw any general conclusions about which areas to improve. Nevertheless, it is important to be aware of which areas that could be an issue in process improvement and historic empirical studies might be a very good indicator on which areas to focus on.

A concrete example that supports the discussion above is the one about synchronization of processes. In the literature, this was not a commonly discussed issue while the studied organization encounters large problems in this area. Therefore, in this organization, it seems to be the area where it is most promising to reach the largest benefits.

The studied organization has a history of developing processes within each department without a common instance for coordinating the improvement work, which has resulted in unsynchronized processes. We do not argue about if this is a good or bad strategy for improving processes, it has both strengths and weaknesses. However, as the results indicate, it seems to be important that even if an organization focuses on developing processes within each department, it is important to coordinate the interfaces of the processes between the departments.

It should however be noted that the key factors are not separate, without interactions between them. Baselining the current way of working is tightly connected with user participation. If no users are involved in when providing a baseline, the baseline will probably not reflect the current way of working. Further, different processes and different persons need baselines of their own processes. If these are not synchronized, they will not work due to unsynchronized interfaces.

In order to get the users of processes to be involved (to baseline and synchronize), management must commit to the process work and provide time and resources for the users. Management must also provide some kind of instance that is responsible for synchronizing the processes. When the processes are documented, the users must also be a part of the development in order to provide an adequate level of the documentation. When all these parts are in place, a good working change management of defined processes must be in place in order to have process descriptions that are not out of date. In this work, management must provide time and resources so that the users of defined processes could be a part of the updating procedure so that the processes are documented and synchronized in a correct way.

The discussion above could probably go on forever. The important thing to remember when conducting process related work is to identify the factors that are in most need for improvement, focus on these, without neglecting to consider what implications this have on the other factors.

\section{Conclusions}

This article presented a study that was conducted at a fairly large company in south Sweden. The study was divided into three different parts: a qualitative part (interviews), a literature part (survey), and a quantitative part (questionnaire). In the article we have presented six different areas that have been identified as key areas for successful process management. These areas are:

- Baselining the current way of working

- Synchronization between processes

- User involvement

- Management commitment

- Change management

- Documentation

In order to determine which factors that were most important and find the key success factors, the three parts of the study have been triangulated. Further, a ranking of the different factors was made from the result of each part of the study.

The result of the ranking showed that baselining the current way of working and synchronization between processes were considered as the most important areas. This does not correspond very well with the results from the literature study conducted. In the literature study, synchronization of processes was considered as the least important part of process management. This indicates that it is hard to generalize results in this area. Historical studies and results 
are a very good point to start from but it is important to consider the individual differences in specific organizations.

The results from this study further indicate that synchronization of processes might be an area where further research ought to be conducted. Due to the fact that this was the least discussed factor within the literature studied and that it was the most important factor in the studied organization, it is an area that might have got too little attention in the past. However, no general conclusions could be drawn from this study. The study just considers one specific case and it is important that further research is conducted in order to find out if other organizations encounter the same problems. If some do, what are the characteristics of such organizations?

Further, the results show that the different factors are very tightly connected. If a successful process improvement programme is to be started, it is not enough to just consider the factors that are most important at the moment, but also other factors that are regarded as important in literature and in other reliable sources.

\section{References}

[1] Marciniak, J. J., Encyclopedia of Software Engineering, Volume 1 A-N, John Wiley \& Sons, New York, United States, 1994.

[2] Gilb, T., Competitive Engineering: A Handbook for Systems and Software Engineering Management Using Planguage, Addison-Wesley, Will be published on January 16, 2004, Book manuscript available from Internet <http://www.result-planning.com> (June 25, 2003).

[3] DeMarco, T., and Lister, T., Peopleware - Productive Projects and Teams, Second Edition, Dorset House Publishing Co, New York, United State, 1999.

[4] Conradi, R. and Dybå, T., “An Empirical Study on the Utility of Formal Routines to Transfer Knowledge and Experience”, Proceedings of the joint 8th European Software Engineering Conference (ESEC) and 9th ACM SIGSOFT International Symposium on the Foundations of Software Engineering (FSE), Vienna, Austria, 10-14 September 2001.

[5] Curtis, B., Kellner, M. I., and Over, J., "Process Modeling”, Communications of the ACM, 35(9), September 1992, pp. 75-90.

[6] Pfleeger, S. L., Software Engineering: Theory and Practice. Prentice Hall, Upper Saddle River, New Jersey, United States, 1998.

[7] Regnell, B., Höst, M., Natt och Dag, J., Beremark, P., Hjelm T., "Visualization of Agreement and Satisfaction in Distributed Prioritization of Market Requirements". 6'th InternationalWorkshop on Requirements Engineering: Foundation for Software Quality, June 5-6 2000, Stockholm, Sweden.
[8] Martella, R. C., Nelson, R., Marchand-Martella, N. E., Research Methods. Allyn \& Bacon, Needham Heights, Massachusetts, United States, 1999.

[9] Humphrey, W. S., Managing Technical People - Innovation, Teamwork, and the Software Process. Addison-Wesley, Massachusetts, United States, 1997.

[10] Olsson, T., and Runeson, P., "Baselining Software Processes as a Starting Point for Research and Improvement”, First Swedish Conference on Software Engineering Research and Practice (SERP), Ronneby, Sweden, 25-26 October 2001.

[11] McFeeley, B., "IDEAL ${ }^{\text {SM }}$ A User's Guide for Software Process Improvement”, Handbook CMU/SEI-96-HB-001, Software Engineering Institute, Carnegie Mellon University, Pittsburgh, 1996.

[12] Ibrahim, R. L., and Hirmanpour, I., "The Subject Matter of Process Improvement: A Topic and Reference Source for Software Engineering Educators and Trainers”, Technical Report CMU/SEI-95-TR-003, Software Engineering Institute, Carnegie Mellon University, Pittsburgh, 1995.

[13] Russo, C. W. R., “12 Rules to Make Your ISO 9000 Documentation Simple and Easy to Use”, ASQC Quality Progress, Vol. 30, March 1997, pp. 51-53.

[14] Becker, U., Hamann, D., and Verlage, M., "Descriptive Modeling of Software Processes”, International Software Engineering Research Network (ISERN) Technical Report ISERN-97-10, Fraunhofer Institute for Experimental Software Engineering, 1997.

[15] ISO 9001 - The TickIT Guide, 2001.

[16] Stelzer, D., and Mellis, W., "Success Factors of Organizational Change in Software Process Improvement”, Software Process-Improvement and Practice, 4 (4), pp. 227-250, 1999.

[17] Humphrey, W. S., Managing the Software Process, AddisonWesley, United States, 1989.

[18] O’Hara, F., “European Experiences with Software Process Improvement”, Proceedings of the International Conference on Software Engineering, Limerick, Ireland, 4-11 June 2000, pp. 635-640.

[19] Paulk, M. C., Weber, C. V., Curtis, B., and Chrissis, M. B., The Capability Maturity Model: Guidelines for Improving the Software Process, Addison-Wesley Publishing Company, United States, 1995.

[20] Kock, N., "Fostering Interdepartmental Knowledge Communication Through Groupware: A Process Improvement Perspective", Proceedings of the International Conference on Supporting Group Work, New York, United States, 1997, pp. 29-37.

[21] Becker-Kornstaedt, U., “Towards Systematic Knowledge Elicitation for Descriptive Software Process Modeling”, Proceedings of the Third International Conference on Product-Focused Software Processes Improvement (PROFES), Lecture Notes in Computer Science 2188, Kaiserslautern, Germany, 10-13 September 2001, pp. 312-325. 